\title{
Robust cancer-specific gene expression by a novel cassette with hTERT and CMV promoter elements
}

\author{
MASAKIYO SAKAGUCHI $^{1 *}$, TAKUYA SADAHIRA ${ }^{2 *}$, HIDEO UEKI $^{2}$, RIE KINOSHITA $^{1}$, \\ HITOSHI MURATA ${ }^{1}$, KEN-ICHI YAMAMOTO ${ }^{1}$, JUNICHIRO FUTAMI ${ }^{3}$, YASUTOMO NASU ${ }^{2}$, \\ KAZUHIKO OCHIAI ${ }^{4}$, HIROMI KUMON ${ }^{5}$, NAM-HO $\mathrm{HUH}^{1}$ and MASAMI WATANABE ${ }^{2,6}$
}

\author{
Departments of ${ }^{1}$ Cell Biology, ${ }^{2}$ Urology, ${ }^{3}$ Medical and Bioengineering Science, Okayama University, Okayama 700-8558; \\ ${ }^{4}$ Department of Veterinary Nursing and Technology, School of Veterinary Science, Nippon Veterinary and Life Science \\ University, Tokyo 180-8602; ${ }^{5}$ Innovation Center Okayama for Nanobio-targeted Therapy, Okayama University \\ Graduate School of Medicine, Dentistry and Pharmaceutical Sciences; ${ }^{6}$ Center for Innovative Clinical Medicine, \\ Okayama University Hospital, Okayama University, Okayama 700-8558, Japan
}

Received September 26, 2016; Accepted February 8, 2017

DOI: $10.3892 / o r .2017 .5710$

\begin{abstract}
We developed and validated a novel hTERT/CMV promoter element-driven gene expression cassette that can robustly enhancecancer-specific geneexpression. The following gene expressional elements were located in tandem within the plasmid construct: [hTERT core promoter, cytomegalovirus (CMV) minimized promoter, RU5' sequence, an inserted gene, BGH polyA, hTERT enhancer]; this is hereafter referred to as the hT/Cm-R-hT construct. Using various human cancer cell lines and normal cells, the cancer-specific transcription of the green fluorescent protein (GFP) gene was examined by western blotting and fluorescence microscopy. Cancer-specific gene expression was robustly achieved in the hT/Cm-R-hT plasmid in comparison to the other control hT/Cm-driven construct. Notably, the expression level of GFP observed in the hT/Cm-RhT-driven construct was superior to that of the control plasmid with the conventional CMV promoter in HEK293 cells, which are known to possess higher hTERT activity than normal cells. We next examined the availability of hT/Cm-R-hT in detecting the target GFP expressing cancer cells from human peripheral blood mononuclear cells (PBMCs). The hT/Cm-R-hT plasmid successfully induced cancer-specific gene expression; the robust expression of GFP was observed in target HeLa cancer cells, whereas GFP was not visibly expressed in normal PBMCs. The plasmid allowed for the selective visualization
\end{abstract}

Correspondence to: Professor Masami Watanabe, Department of Urology, Okayama University, 2-5-1 Shikata-cho, Okayama 700-8558, Japan

E-mail: mwcorrespondence@gmail.com

*Contributed equally

Key words: gene expression, cancer, hTERT, cytomegalovirus promoter, enhancer of viable HeLa cancer cells in mixed cell cultures containing up to 10000-fold more PBMCs. These findings indicate that the hT/Cm-R-hT expressional system is a valuable tool for detecting viable cancer cells mixed with normal cells. The current system can therefore be applied to the in vitro detection of cancer cells that are disseminated in the blood and other types of body fluid in vivo. Since the current system can also be applied to other types of vectors, including virus vectors, this approach using the hTERT promoter-based construct is expected to become a valuable tool for enhancing cancerspecific gene expression.

\section{Introduction}

Telomeres are specific sequences that are composed of tandem repeats of the TTAGGG at the end of human chromosomes (1). The possible functions of the non-coding DNA include the prevention of chromosome degradation, end-to-end fusion, rearrangements and chromosome loss (2). In normal cells, telomere shortening occurs with each cellular division. Telomerase is a reverse transcriptase enzyme that is composed of a catalytic component, telomerase RNA and a human telomerase reverse transcriptase (hTERT) component (3). Telomerase activity is undetectable in the majority of normal human somatic cells. However, telomerase activity is maintained in stem/progenitor cells in self-renewing tissues (4). In addition, telomerase activation is observed in almost $90 \%$ of human cancers, indicating that telomerase plays an important role in cancer development (5).

Various transcriptional targeting strategies using different tissue-specific promoters have been tested. The hTERT promoter has been well characterized and has been shown to promote the cancer cell-specific gene expression in a broad range of malignancies (6-9). The hTERT promoter is widely applied in cancer-specific gene therapy and imaging $(10,11)$. However, the use of the hTERT promoter is often limited because its driving ability as a specific promoter is weak. Consequently, the expression levels of the reporter gene and 
therapeutic genes are insufficient (12-14). Thus, the modification of the hTERT promoter-driven gene expression cassette is necessary for enhancing the cancer-specific gene expression.

Gene expression promoters, such as the CMV (cytomegalovirus), RSV (Rous sarcoma virus) and SV40 (simian virus 40) promoters, have been used to increase the expression of genes in a variety of normal and cancer cells, and have achieved good transduction efficiency (15). Since the minimal element of a viral promoter is often used as a basic transcriptional unit (16), we modified the hTERT promoter by adding the CMV minimized promoter and constructed a series of chimeric promoter-driven gene expression cassettes. In the present study, we evaluated the extent to which the novel cassettes enhanced gene expression and the degree of hTERT promoter-dependency in various human cells.

\section{Materials and methods}

Cell culture. The following human cell lines were obtained from the American Type Culture Collection (ATCC, Rockville, MD, USA) if not notified: HEK293 (human embryonic kidney cell line), HeLa (cervical cancer), PC3 (prostate adenocarcinoma), HepG2 (hepatocellular carcinoma), Hep3B (hepatocellular carcinoma), HCT116 (colon cancer), RPTEC (normal renal proximal tubule cells; Lonza, Basel, Switzerland), UMUC1 (bladder cancer), UMUC3 (bladder cancer), HT1197 (bladder cancer), RT4 (bladder cancer), MCF7 (mammary gland adenocarcinoma), OUMS-24 (normal human fibroblasts; kindly provided by Dr Masayoshi Namba), NHK (normal human keratinocytes; Kurabo Industries Ltd., Osaka, Japan) and human iPS (induced pluripotent stem) cells (Riken $\mathrm{BCR}$, Tsukuba, Japan). These cell lines were cultivated in $\mathrm{D} / \mathrm{F}$ medium (Invitrogen, Carlsbad, CA, USA) supplemented with $10 \%$ FBS or cultivated using the medium recommended by the supplier. The human iPS cells were cultured and maintained as previously described (17).

Construction of the plasmid vectors. We constructed new plasmids in which the promoter consisted of the hTERT core promoter and the CMV minimized promoter (hT/Cm). The series of modifications of the hT/Cm promoter-driven construct are shown in Fig. 1B. They were performed in the pDNR-1r promoter-less vector (Takara Clontech, Mountain View, CA, USA). We finally developed a (hT/Cm-R-hT) plasmid construct in which the following gene expression elements were located in tandem: [hTERT core promoter, CMV minimized promoter, RU5' sequence (R), an inserted gene, BGH polyA, hTERT enhancer (hT)] (Fig. 1B). The R segment and part of the U5 sequence (RU5'), BGH (bovine growth hormone) polyadenylation (polyA) signal and the sequences of multiple cloning sites were the same as those of previous reports $(15,18)$. The hTERT promoter element [189 bp: Accession no. DQ264729 (1618-1806)] was used and inserted at either the 5'-side alone or at both the 5'- and 3'-side of the cDNA in the constructs. The minimal CMV sequence that was used in the present study was as follows: GGTAGGCGTGTACGGTGGGAGGCCTATA TAAGCAGAGCTCGTTTAGTGAACCGTCAGATCGCCT GGAGACGCCATCCACGCTGTTTTGACCTCCATAGAA GACACCGGGACCGATCCAGCCTCCGCGGCCCCGCA TTCGAGCTCGGTACCCGG.

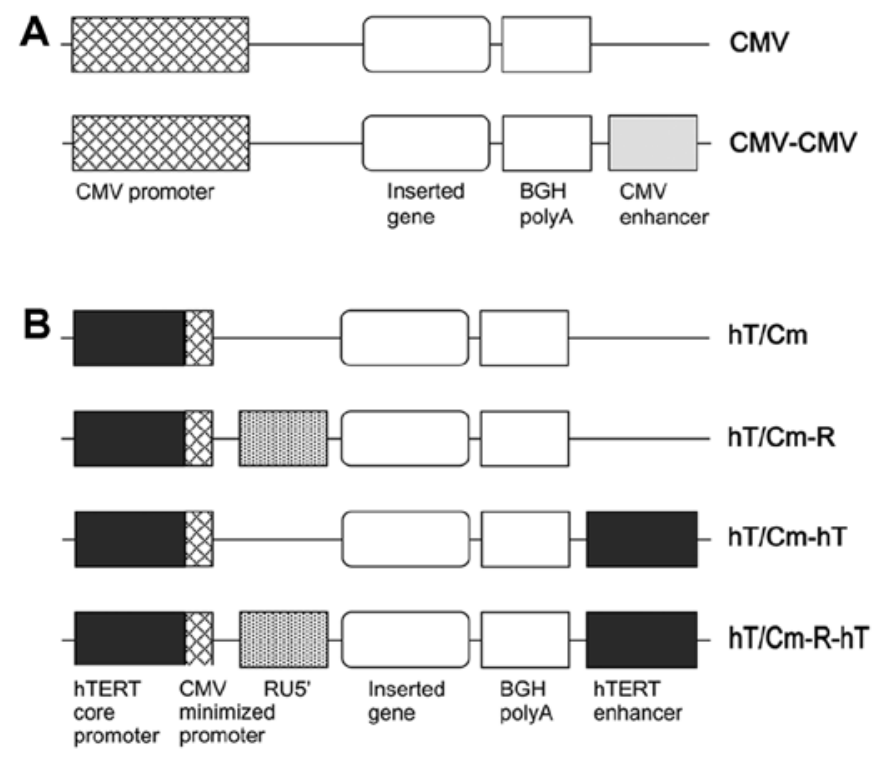

Figure 1. A schematic diagram of the expressional plasmid constructs. (A) We added the CMV enhancer to the end of the conventional (CMV) construct in our previously developed (CMV-CMV) construct in order to enhance CMV promoter-dependent transcription. (B) The series of modifications of the $\mathrm{hT} / \mathrm{Cm}$ promoter-driven construct are shown. The basic promoter consists of the hTERT core promoter and the CMV minimized promoter $(\mathrm{hT} / \mathrm{Cm})$. We finally developed the (hT/Cm-R-hT) plasmid construct, in which the following gene expressional elements were located in tandem: [hTERT core promoter, CMV minimized promoter, RU5' sequence (R), an inserted gene, BGH polyA, hTERT enhancer (hT)].

We previously developed the (CMV-CMV) plasmid construct (Fig. 1A) by adding the CMV enhancer to the end of the conventional (CMV) construct, which enhanced CMV promoter-dependent transcription (18). The full-length cDNAs of green fluorescence protein (GFP) from the vector pEGFP-N2 (Takara Clontech), red fluorescence protein (DsRed) from the vector pDsRed-Express (Takara Clontech), glutathione-Stransferase (GST) from the vector pGEX6P1 (GE Healthcare Life Sciences, Tokyo, Japan), and luciferase from the vector pGL4.14 (luc2/Hygro) (Promega BioSciences, San Luis Obispo, CA, USA) were amplified and inserted into the newly designed constructs. An advanced two-step transcriptional amplification (ad-TSTA) system, which has been previously reported, was able to enhance cancer-specific hTERT promoter-driven transcription (13).

Cell transfections and assays. The cells were plated in a culture medium in 6-well or 24-well plates. After $24 \mathrm{~h}$, the transient transfection of the GFP-, luciferase- or indicated gene encoded in the CMV, CMV-CMV, hT/Cm, hT/Cm-R, hT/Cm-hT, or $\mathrm{hT} / \mathrm{Cm}-\mathrm{R}-\mathrm{hT}$ plasmid was performed using the Lipofectamine transfection reagent (Invitrogen). At $24 \mathrm{~h}$ after transfection, the expression of GFP was analyzed by fluorescence microscopy or western blotting. The luciferase expression assay was performed as previously described $(13,14)$. Briefly, the effector plasmid was co-transfected with the reporter plasmid derived from the dual-reporter luciferase assay kit (Promega, Madison, WI, USA). After $48 \mathrm{~h}$ of incubation, the cells were harvested and their luciferase activity was determined using a luciferase assay kit and a luminescence microplate reader, according to the manufacturer's instructions. 
A

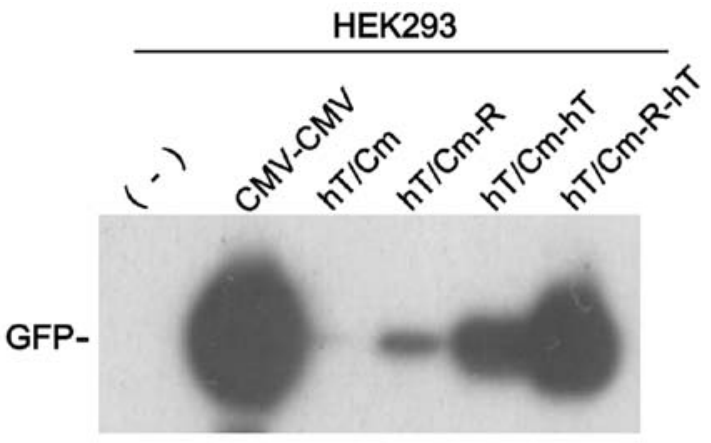

B

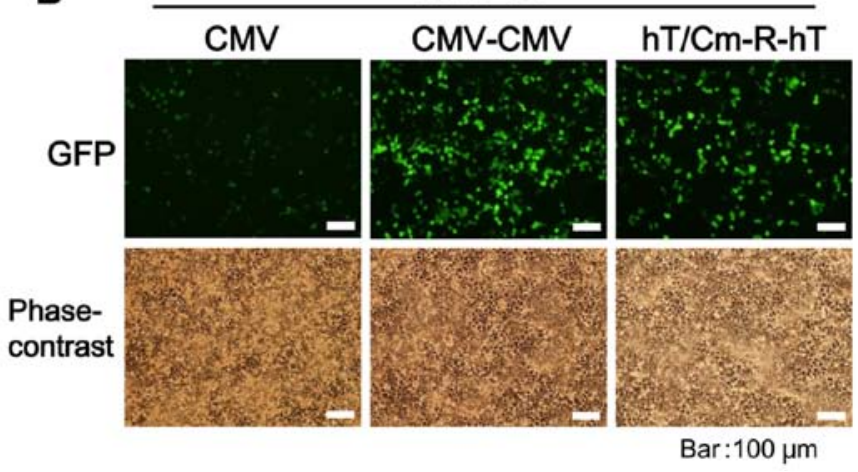

Figure 2. The GFP expression of the plasmid constructs was examined in HEK293 cells. (A) GFP expression levels of the CMV-CMV and $\mathrm{hT} / \mathrm{Cm}$-based constructs were analyzed by western blotting. (B) The GFP expression of the CMV-based and hT/Cm-R-hT constructs was examined by fluorescence microscopy.

Western blotting. The cells were harvested at $24 \mathrm{~h}$ after transfection with the expression vectors and were subjected to SDS-PAGE followed by western blotting, as previously described (15). Goat anti-schistosomal GST antibody (GE Healthcare Life Sciences), rabbit anti-GFP antibody (Takara Clontech), rabbit anti-DsRed antibody (Takara Clontech), rabbit anti-telomerase (Abcam Inc., Cambridge, MA, USA) and mouse anti-tubulin (Sigma, St. Louis, MO, USA) were used as the primary antibodies.

TERT activity assays. A real-time QRT-PCR for hTERT was performed as previously described and the results were estimated as the cellular endogenous TERT activity $(13,14)$. The primers used for the real-time PCR were TERT-F', 5'-CCAT CAGAGCCAGTCTCACCTTC-3', TERT-R', 5'-ACCGTCTG GAGGCTGTTCA-3'; glyceraldehyde-3-phosphatedehydrogenase (GAPDH)-F', 5'-GCACCGTCAAGGCTGAGAAC-3', GAPDH-R' and 5'-TGGTGAAGACGCCAGTGGA-3'.

In vitro detection of viable cancer cells with the $h T / C m-R-h T$ plasmid. The experimental system for the in vitro detection of GFP-expressing HeLa cancer cells is shown in Fig. 6A. The target HeLa cancer cells were pretreated with CellTracker Orange (Invitrogen) as a tracer according to the manufacturer's instructions. The cancer cells were then trypsinized and mixed with human peripheral blood mononuclear cells (PBMCs) at the indicated target frequency ratios (14). The mixed cells were transfected with the hT/Cm- or hT/Cm-RhT-GFP plasmid. After $24 \mathrm{~h}$, the GFP expression of the cells

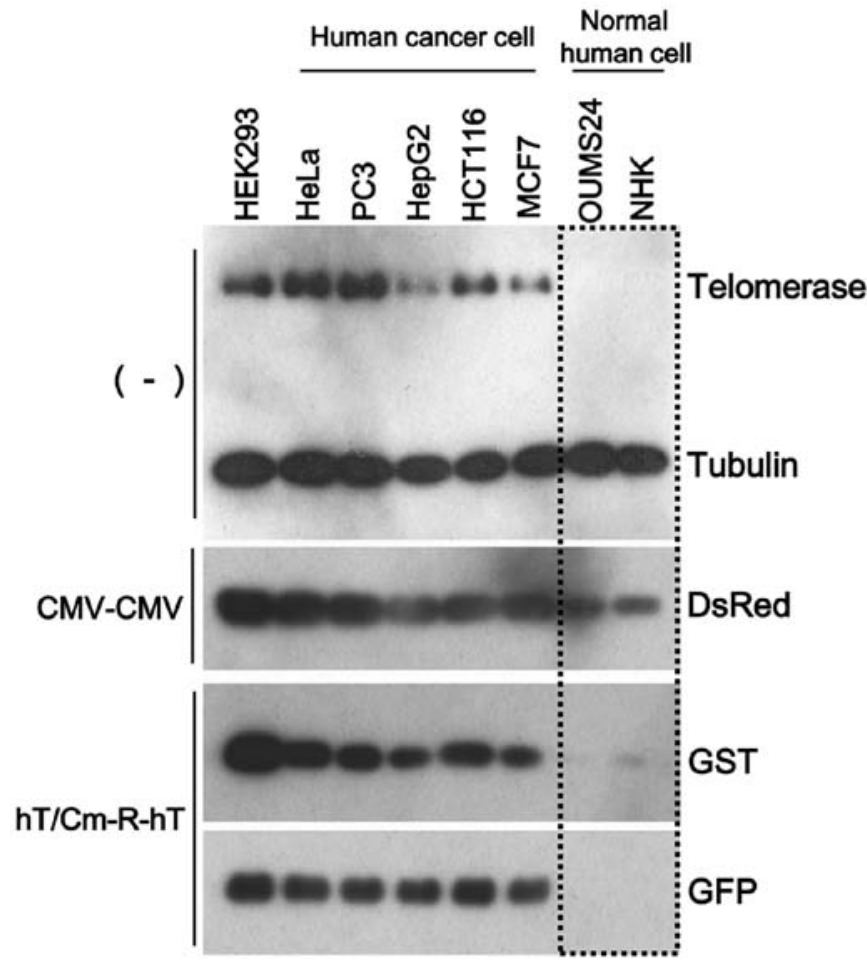

Figure 3. hTERT promoter-dependent cancer-specific gene expression by the $\mathrm{hT} / \mathrm{Cm}-\mathrm{R}-\mathrm{hT}$ plasmid constructs was examined by western blotting in human cancer cell and normal (non-cancer) cell lines. The upper panel shows the intrinsic gene expression of telomerase and tubulin. The cancer-specificity of the hT/Cm-R-hT plasmids (shown in lower panel) was compared to that of the CMV-CMV plasmid (middle panel).

was examined and CellTracker Orange staining was observed by fluorescence microscopy.

Statistical analysis. The data are presented as the mean \pm standard deviation. A regression analysis was performed to examine the correlation between 2 parameters. P-values of $<0.05$ were considered to indicate statistically significant differences.

\section{Results}

$h T / C m-R-h T$ system achieves powerful gene expression. We previously reported a novel gene expression construct that enables the enhancement of the expression level of a cargo gene with the structural modification of the vectors $(15,18)$. The key to the modification is the insertion of a certain enhancer (such as a CMV or hTERT enhancer) just behind the polyadenylation (polyA) signal sequence and the insertion of the RU5' sequence, which enhances the transcription efficiency. Based on the findings, we constructed four hT/Cm promoter-based plasmids (Fig. 1B) and compared them in vitro gene expression ability. The GFP gene and HEK 293 cells were used for this assessment. Western blot analysis of the series of modifications revealed that the expression of GFP was strongest in hT/Cm-R-hT plasmid (Fig. 2A). The expression of GFP by the $\mathrm{hT} / \mathrm{Cm}-\mathrm{hT}$ plasmid was superior to that of the $\mathrm{hT} / \mathrm{Cm}$ promoter alone and the hT/Cm-R plasmid, indicating that the insertion of the hTERT enhancer at the 3'-side of the cDNA markedly enhances the expression of the cargo gene. Under fluorescence microscopy, the hT/Cm-R-hT plasmid system showed 


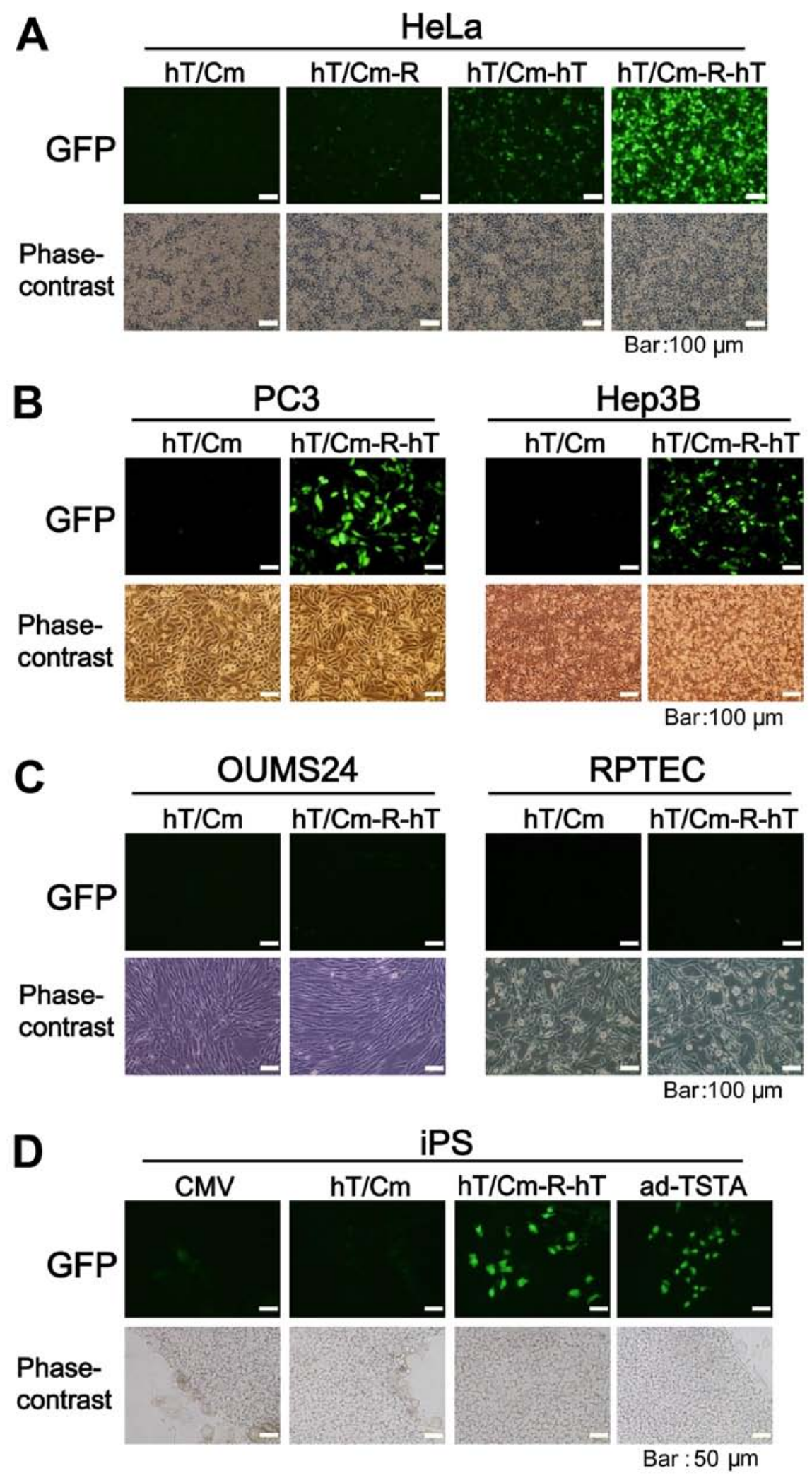

Figure 4. Robust cancer-specific expression of GFP by the hT/Cm-R-hT plasmid was examined in human cancer (A and B), normal (non-cancer) (C) and iPS (induced pluripotent stem) (D) cell lines. The advanced two-step transcriptional amplification (ad-TSTA) gene expressional cassette is a plasmid construct that contains TSTA system that we previously developed. Fluorescence microscopy was performed to assess the GFP expression of the cells.

GFP expression more strongly than the conventional CMV construct (Fig. 2B). Notably, the robust expression that was observed in the hT/Cm-R-hT construct was almost comparable to the strongest CMV-CMV construct (Fig. 2A and B). $h T / C m-R-h T$ system achieves robust cancer-specific gene expression. To further confirm the sophistication of the $\mathrm{hT} / \mathrm{Cm}-\mathrm{R}-\mathrm{hT}$ construct in cancer-specific gene expression, we added normal human cells for the in vitro analyses of 

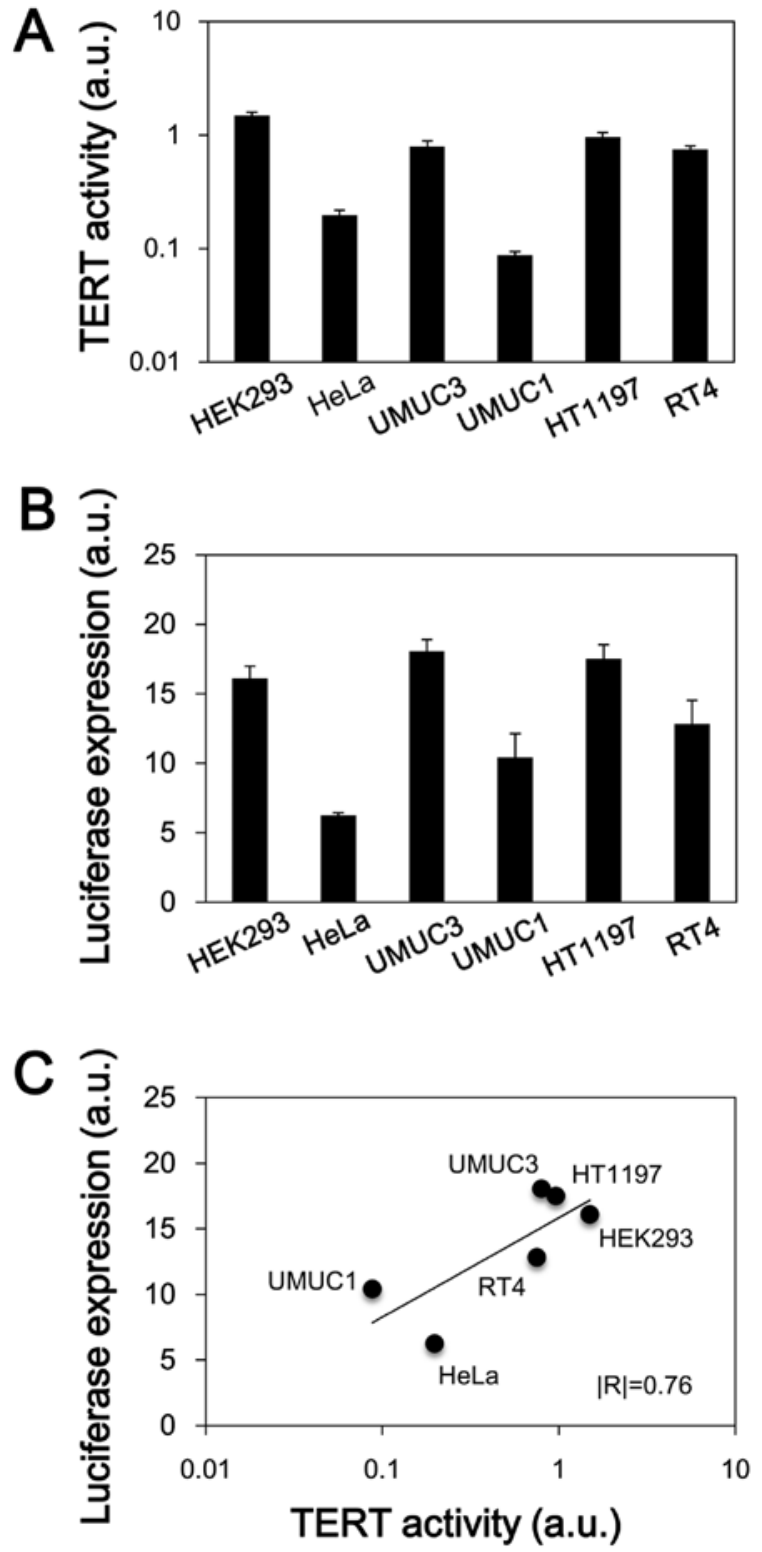

Figure 5. The hTERT promoter-dependent gene expression of the $\mathrm{hT} / \mathrm{Cm}-\mathrm{R}-\mathrm{hT}$ plasmid construct was confirmed by analyzing its correlation with the cellular TERT activity. (A) The TERT activity of a variety of human cell lines was analyzed. A real-time QRT-PCR was performed to estimate the TERT activity. The data are shown as arbitrary units (a.u.) and were obtained from triplicate measurements. (B) The luciferase expression (a.u.) of the $\mathrm{hT} / \mathrm{Cm}-\mathrm{R}-\mathrm{hT}$ plasmid was analyzed in a variety of human cell lines. The level of luciferase expression was estimated based on the luminescence of luciferin, a substrate for luciferase. (C) A regression analysis was performed to examine the relationship between the TERT activity (a.u.) and luciferase expression (a.u.) induced by the $\mathrm{hT} / \mathrm{Cm}-\mathrm{R}-\mathrm{hT}$ system. The analysis indicated a strong association between the two parameters.

gene expression. We performed simultaneous triple transfection using three types of reporter gene-expressing plasmids (CMV-CMV plasmid encoding DsRed, hT/Cm-R-hT plasmids encoding GST or GFP). The CMV-CMV plasmid, which works in a broad range of cell types, including normal cells, was used for the negative control for the cancer-specific gene expression. After the transfection of these constructs to the indicated cells, we found that DsRed was expressed in all cells, including the normal cells, while GST and GFP were expressed at almost undetectable levels in the normal cells, with the expression restricted to the cancer cells (Fig. 3). Thus, the hT/Cm-R-hT construct possessed the ability of cancer-specific gene expression. Consistent results were obtained in various normal and cancer cells under fluorescence microscopy (Fig. 4A-C), showing that the plasmid enhanced the expression and that the cancer-specific enhancement was not lost. In this experimental condition, the expression of GFP was relatively strong in human iPS cells, which are known for tumorigenicity, in comparison to normal cells (Fig. 4C and D). The hTERT promoter-driven ad-TSTA plasmid, the gene expression is also restricted in cancer cells $(13,14)$, was tested using iPS cells and similar results were obtained (Fig. 4D).

hTERT-dependent gene expression of the hT/Cm-R-hT system in human cancer cells. We examined whether the newly developed hT/Cm-R-hT construct exhibits hTERT promoterdependent gene expression. The endogenous TERT activity was first determined in each of the cell lines that was used in this assessment (Fig. 5A). HEK293, UMUC3, HT1197 and RT4 cells showed relatively higher activity levels in comparison to the other cells. We next examined the expression of the luciferase reporter gene in the hT/Cm-R-hT construct (Fig. 5B). The expression of luciferase tended to be very similar to the TERT activity in the cell lines (Fig. 5A and B). In a regression analysis, a strong positive correlation $(|R|=0.76)$ was confirmed between the TERT activity and the luciferase expression level (Fig. 5C); however, the result did not reach statistical significance $(\mathrm{P}=0.08)$. This result reveals that the gene expression of the hT/Cm-R-hT system is TERT activity-dependent, which indicates that it is hTERT promoter-dependent. These findings were consistent with the western blot results, which revealed that the hT/Cm-R-hT plasmid achieved cancer-specific gene expression depending on the endogenous telomerase expression levels, indicating the levels of TERT activity (Fig. 3).

In vitro detection of viable cancer cells using $h T / C m-R$ $h T$-GFP plasmid system. We next evaluated the utility of the hT/Cm-R-hT plasmid system in the in vitro detection of viable cancer cells (Fig. 6A). HeLa cancer cells were mixed with PBMCs and the cells were transfected with the hT/Cm-R-hT-GFP plasmid. The GFP expression of these cells was examined under fluorescence microscopy. HeLa cancer cells were pretreated with the CellTracker Orange as a tracer to ensure that the cells expressing the GFP signals were indeed the target HeLa cancer cells. Dual fluorescence microscopy confirmed that most of GFP-positive cultured cells were HeLa cancer cells that had been labeled with CellTracker Orange at different target frequency ratios (1:10, 1:1000 and 1:10000) (Fig. 6B). The fact that HeLa cancer cells could be morphologically distinguished from the other PBMCs in the bright field reinforced the accuracy of the experiments.

\section{Discussion}

The success of gene therapies and imaging approaches using the cancer-specific gene expression is influenced by the expression levels of gene products. Although the hTERT promoter has been well-characterized as a broad range cancerspecific promoter $(6,9)$, the gene expression driven by the hTERT promoter is often weak due to its poor transcriptional 
A

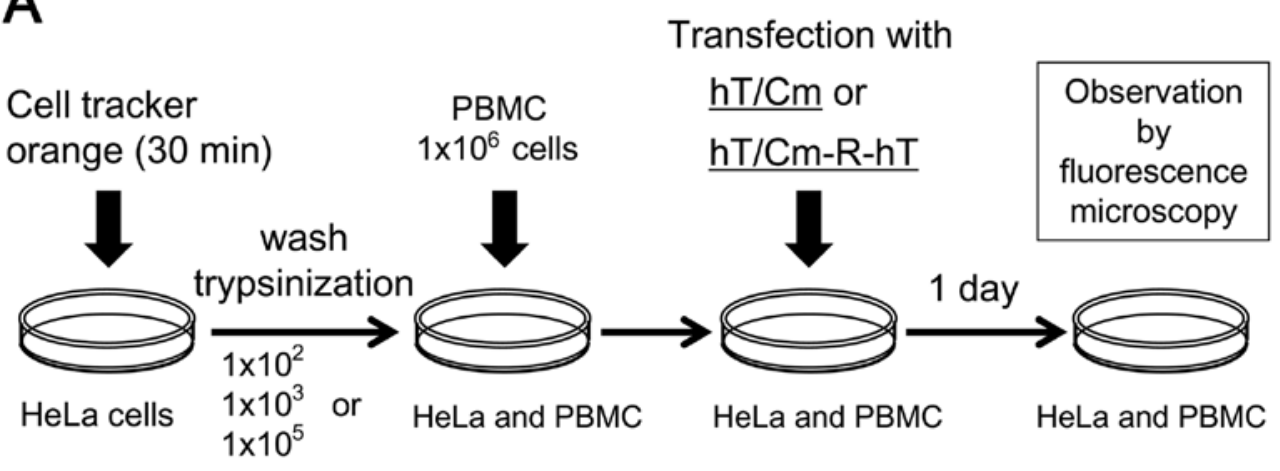

B

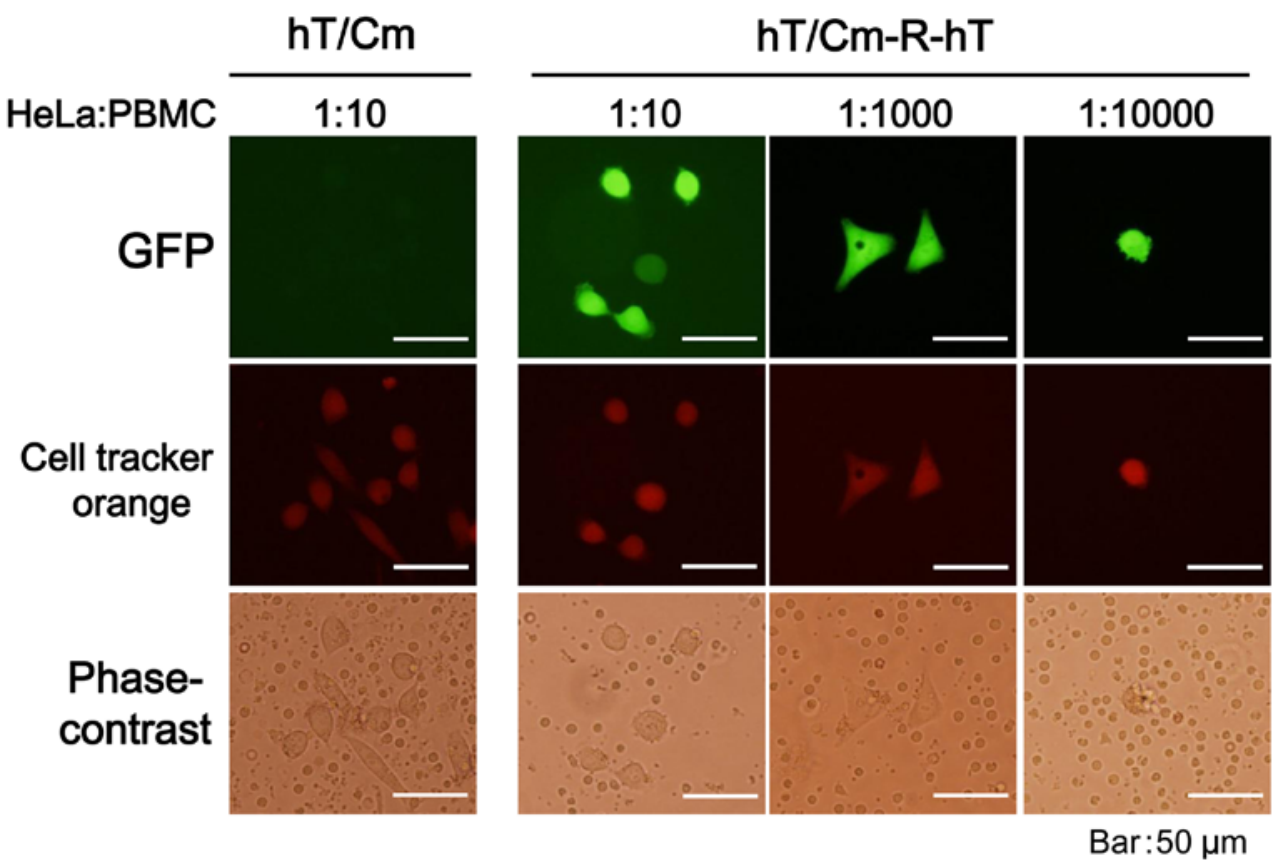

Figure 6. Cancer-specific visualization using the hT/Cm-R-hT-GFP plasmid. (A) The selective visualization of HeLa cancer cells using the hT/Cm- or hT/Cm-RhT-GFP plasmid. A schematic illustration of the experimental procedure is shown. The HeLa cancer cells were pretreated with the CellTracker Orange as a tracer to determine whether the cells expressing GFP signals were the target HeLa cancer cells. The target HeLa cancer cells were mixed with peripheral blood mononuclear cells (PBMCs) and both GFP and the tracer were directly examined by fluorescence microscopy. (B) Representative images of the co-cultured cells (HeLa cancer cells and PBMCs) are shown. HeLa cancer cells were mixed in the PBMCs at the indicated frequency (1:10, 1:1000, and 1:10000) and were confirmed to be HeLa cancer cells when both GFP expression and CellTracker Orange were confirmed under fluorescence microscopy. The use of the hT/Cm-R-hT-GFP plasmid allowed for the target HeLa cancer cells to be clearly identified among the PBMCs.

activity $(13,14)$. On the other hand, the combination of a promoter and an enhancer has been attempted in our previous studies, with several showing improved gene transcription $(15,18)$. For the purpose of overcoming the weakness of the hTERT promoter, we herein developed a novel hT/Cm-R-hT construct. We demonstrated that the plasmid robustly enhances the hTERT promoter-driven cancer-specific gene expression. Based on the cancer-specificity, we also confirmed the availability of this cassette for the in vitro imaging and detection of human cancer cells mixed with normal human cells.

In the current gene expression system, we used a chimeric promoter element derived from the hTERT and CMV promoters upstream of the cargo gene. When the hT/Cm promoter was combined with the subsequent RU5' sequence, an inserted gene, BGH polyA, and the hTERT enhancer, the expression of GFP was significantly enhanced to a level that was nearly comparable to the CMV-CMV plasmid construct, which is one of the strongest expression systems that we reported (18). Furthermore, as the TERT activity was elevated in multiple cancer cell lines, the luciferase gene expression of the hT/Cm-R-hT system was stronger. These results indicate that this system is advantageous in the cancer cells with higher TERT activity in terms of the gene expression.

Importantly, we were able to use the hT/Cm-R-hT-GFP plasmid to examine the fluorescence imaging of the promoter-driven human cancer cells by microscopy. The hT/Cm-R-hT-GFP plasmid successfully induced cancerspecific gene expression, showing the robust expression of GFP in the target cancer cells, but no visible expression of GFP in PBMCs. Viable human cancer cells were selectively visualized in cultured cells containing a mixture of 10-, 1000-, and 10000 -fold more PBMCs. An ideal diagnostic agent for cancer would be able to selectively target tumor cells. The present study indicates that the hT/Cm-R-hT system may be useful 
for detection of target cancer cells. The current system can be applied to the in vitro detection of cancer cells disseminated in vivo in the blood and in other types of body fluid.

We also tried to assess our vector in iPS cells. The human iPS cells are invaluable for therapeutic approaches owing to their pluripotency except for the high ability of tumor formation in vivo. One of the reasons of tumorigenicity comes from marked expression of hTERT in iPS cells (19). This leads to our expectation that our newly developed vector is able to detect not only cancer cells but also in vivo tumor-initiating cells, such as iPS cells. As expected, the expression of GFP was relatively strong in human iPS cells in comparison to normal cells. These results suggest that our innovative vector has an advantage to distinguish tumor-initiating cells from normal cells with significant sensitivity. In addition to this, we also considered that the vector is useful to assess stem cells and differentiated cells in the iPS population.

Since the current system can also be applied to other types of vectors, such as virus vectors, this approach is widely expected to become a valuable tool for enhancing the hTERT promoter-dependent cancer-specific gene expression. It would be of interest to extend this strategy to clinical practice and to confirm the efficacy of the system as a non-invasive method for diagnosing and evaluating various types of cancer.

\section{Acknowledgements}

This work was supported by scientific research grants (JSPS KAKENHI grant nos.: JP16K11004, JP15H04297, JP15H04974) from the Ministry of Education, Culture, Sports, Science and Technology of Japan and supported by a grant for promotion of science and technology in Okayama prefecture (by MEXT) 'Creation of nanobiotargeted therapy using REIC as a therapeutic gene for cancer'. The authors would like to thank Ms. Fusaka Oonari and Ms. Shun-Ai Li (Okayama University) for their valuable assistance. Okayama University and Momotaro-Gene Inc. are applying for patents on the gene expression systems of the CMV-CMV and hT/Cm-based constructs that were described in the present study. Drs M.S., Y.N., H.K., N.H.H. and M.W. own stock in Momotaro-Gene Inc.

\section{References}

1. Greider CW: Telomere length regulation. Annu Rev Biochem 65: 337-365, 1996.

2. Greider CW: Chromosome first aid. Cell 67: 645-647, 1991.

3. Nakamura TM and Cech TR: Reversing time: Origin of telomerase. Cell 92: 587-590, 1998.

4. Tahara H, Yasui W, Tahara E, Fujimoto J, Ito K, Tamai K, Nakayama J, Ishikawa F, Tahara E and Ide T: Immunohistochemical detection of human telomerase catalytic component, hTERT, in human colorectal tumor and non-tumor tissue sections. Oncogene 18: 1561-1567, 1999.
5. Kim NW, Piatyszek MA, Prowse KR, Harley CB, West MD, Ho PL, Coviello GM, Wright WE, Weinrich SL and Shay JW: Specific association of human telomerase activity with immortal cells and cancer. Science 266: 2011-2015, 1994.

6. Takakura M, Kyo S, Kanaya T, Hirano H, Takeda J, Yutsudo M and Inoue M: Cloning of human telomerase catalytic subunit (hTERT) gene promoter and identification of proximal core promoter sequences essential for transcriptional activation in immortalized and cancer cells. Cancer Res 59: 551-557, 1999.

7. Kishimoto H, Kojima T, Watanabe Y, Kagawa S, Fujiwara T, Uno F, Teraishi F, Kyo S, Mizuguchi $\mathrm{H}$, Hashimoto Y, et al: In vivo imaging of lymph node metastasis with telomerasespecific replication-selective adenovirus. Nat Med 12: 1213-1219, 2006.

8. Maida Y, Kyo S, Sakaguchi J, Mizumoto Y, Hashimoto M, Mori N, Ikoma T, Nakamura M, Takakura M, Urata Y, et al: Diagnostic potential and limitation of imaging cancer cells in cytological samples using telomerase-specific replicative adenovirus. Int J Oncol 34: 1549-1556, 2009.

9. Cong YS, Wen J and Bacchetti S: The human telomerase catalytic subunit hTERT: Organization of the gene and characterization of the promoter. Hum Mol Genet 8: 137-142, 1999.

10. Huang P, Kaku H, Chen J, Kashiwakura Y, Saika T, Nasu Y, Urata Y, Fujiwara T, Watanabe M and Kumon H: Potent antitumor effects of combined therapy with a telomerase-specific, replication-competent adenovirus (OBP-301) and IL-2 in a mouse model of renal cell carcinoma. Cancer Gene Ther 17: 484-491, 2010

11. Kojima T, Hashimoto Y, Watanabe Y, Kagawa S, Uno F, Kuroda S, Tazawa H, Kyo S, Mizuguchi H, Urata Y, et al: A simple biological imaging system for detecting viable human circulating tumor cells. J Clin Invest 119: 3172-3181, 2009.

12. Iyer M, Wu L, Carey M, Wang Y, Smallwood A and Gambhir SS: Two-step transcriptional amplification as a method for imaging reporter gene expression using weak promoters. Proc Natl Acad Sci USA 98: 14595-14600, 2001.

13. Watanabe M, Ueki H, Ochiai K, Huang P, Kobayashi Y, Nasu Y, Sasaki K, Kaku H, Kashiwakura Y and Kumon H: Advanced two-step transcriptional amplification as a novel method for cancer-specific gene expression and imaging. Oncol Rep 26: 769-775, 2011.

14. Ueki H, Watanabe M, Kaku H, Huang P, Li SA, Ochiai K, Hirata T, Noguchi $\mathrm{H}$, Yamada $\mathrm{H}$, Takei K, et al: A novel gene expression system for detecting viable bladder cancer cells. Int J Oncol 41: 135-140, 2012.

15. Watanabe M, Sakaguchi M, Kinoshita R, Kaku H, Ariyoshi Y, Ueki H, Tanimoto R, Ebara S, Ochiai K, Futami J, et al: A novel gene expression system strongly enhances the anticancer effects of a REIC/Dkk-3-encoding adenoviral vector. Oncol Rep 31: 1089-1095, 2014.

16. Agha-Mohammadi S, O'Malley M, Etemad A, Wang Z, Xiao X and Lotze MT: Second-generation tetracycline-regulatable promoter: Repositioned tet operator elements optimize transactivator synergy while shorter minimal promoter offers tight basal leakiness. J Gene Med 6: 817-828, 2004.

17. Senju S, Haruta M, Matsumura K, Matsunaga Y, Fukushima S, Ikeda T, Takamatsu K, Irie A and Nishimura Y: Generation of dendritic cells and macrophages from human induced pluripotent stem cells aiming at cell therapy. Gene Ther 18: 874-883, 2011.

18. Sakaguchi M, Watanabe M, Kinoshita R, Kaku H, Ueki H, Futami J, Murata H, Inoue Y, Li SA, Huang P, et al: Dramatic increase in expression of a transgene by insertion of promoters downstream of the cargo gene. Mol Biotechnol 56: 621-630, 2014.

19. Huang J, Wang F, Okuka M, Liu N, Ji G, Ye X, Zuo B, Li M, Liang P, Ge WW, et al: Association of telomere length with authentic pluripotency of ES/iPS cells. Cell Res 21: 779-792, 2011. 